\title{
The Relationship between Self Harm, Shame and Guilt: a review
}

\author{
N Navya ${ }^{1}$, Vikas Sharma ${ }^{2}$, Manpreet $\mathrm{Ola}^{3}$ \\ ${ }^{1}$ Research Scholar, Amity University, Haryana \\ ${ }^{2}$ Head of Department, AICP, Amity University, Haryana \\ ${ }^{3}$ Assistant Professor, AICP, Amity University, Haryana \\ Corresponding Author: Manpreet Ola
}

\begin{abstract}
Self-harm is a major health concern behaviour associated with suicide risk and significant psychological distress. Shame and guilt are the moral emotions which play a potential role in the self-harm or self-injury. Theories also suggest that aversive emotional states are an important process that drives the self-harm behaviour. The present review therefore sought to provide a systematic review of the relationship between self-harm, guilt and shame. Most of the article indicates that most forms of shame were associated with selfharm or self-injury. Results of this review support the link between shame and self-harm. Clinically, consideration needs to be given to the role of shame amongst individuals who present self-harm behaviour and more research should be done on determining the relationship between guilt and self-harm behaviour.
\end{abstract}

Keywords: Self harm, guilt, shame, systematic review, suicide.

(Paper received $-20^{\text {th }}$ January 2020 , Peer review completed $-2^{\text {nd }}$ February 2020)

(Accepted $-4^{\text {th }}$ February 2020)

\section{INTRODUCTION}

Due to high prevalence and correlation with suicide risk, self-harm poses a significant health concern. In India, the prevalence rate is as high as $17 \%$ especially among adolescents [1]. Self-harm refers to the deliberate destruction or damage to one's own body tissue, irrespective of suicidal intent and behaviours includes over dosage of medicine, cutting, burning, banging, hitting and scratching oneself. Self-harm behaviour therefore includes suicidal behaviour and non-suicidal self-injury. Recent studies suggest that one of the most common reported reasons for self-harm is around coping with or regulating difficult emotional states. For better understanding the mechanism involving exposure to and regulation of emotional states appear to key to understanding self-harm behaviour. Certain emotions appear especially important in understanding self-harm behaviour. The current review focuses on two such emotions: shame and guilt.

Shame is defined as a cognitive affective construct, comprising negative judgment of the self. The judgment of the self is undesirable and characterized by an evaluation of the self as inherently flawed, inadequate or bad [2]. Guilt on other hand is concerned with one's behaviour and the negative evaluation. Hence, focus is something done by the individual that is perceived as bad or wrong, rather than the evaluating individual themselves. Shame and guilt have been described as "moral" emotions of self-consciousness that arise in response to self-evaluation.

Shame centres upon the individual's perception of themselves in a negative manner. Some researchers have explained two types of shame i.e. external shame which means the individual's perception of being negatively judged by others and whereas, in internal shame individual's judge oneself negatively. In contrast, guilt centres upon the individual's work as bad or wrong and is judged by oneself unlike like shame. A range of psychometric measures have been developed to study various aspects of shame but there are no 
such psychometric measures which measures various components of guilt so far. Shame and guilt can be experienced as emotional states that are unwanted or aversive. Nevertheless, literature suggests that shame may be especially pernicious because of its close ties to the individual's sense of self. Nonetheless, shame is closely linked to different psychological conditions, including anxiety, borderline personality disorder, depression post-traumatic stress disorder (PTSD), and eating disorders. In a population of people with borderline personality disorder (BPD), Brown and others examined the effect of shame on self-inflicted injury. Individuals with BPD are at very high risk of suicidal and non-suicidal self-inflicted injury [3].

Shame and guilt both tend to relate differently to a variety of outcomes. For example, for a variety of behaviours associated with self-harm behaviour, shame and guilt have shown to be risk and protective factors, respectively. It encompasses both externalizing behaviours such as aggression and drug use, as well as internalizing behaviours such as various forms of psychopathology [4]. A sense of shame occurs when one thinks that others are judging them negatively [4]. Researchers concluded in their study of suicidal studies that shame is a better predictor of suicide than guilt [5].

Guilt feelings are likely to serve as a protective mechanism from engaging in self-injurious behaviour, as just that, the guilt-prone individual will correctly judge a negative situation. As their shame prone counterparts may tend to do, they will be less inclined to assess themselves as negative. Guilt can even serve as a protective factor because it is linked to proactive attempts to solve a problem. On the other hand, guilt which is linked to defence, separation and distance.

Shame may also aggravate the effect of high emotional dysregulation, resulting in higher self-harm rates than those with lower levels of shame. An especially risky combination can be the product of being high in both emotional dysregulation and remorse. In comparison, guilt can function as a protective variable against self-harm, whereby guilt correlates with emotional dysregulation to result in lower self-harm rates with higher guilt levels than those with lower guilt levels. Getting high in guilt will buffer the harmful effects of emotional dysregulation on self-harm

The current article aims to provide a systematic review of the available literature pertaining to self-harm and its relationship with shame and guilt. Shame and guilt correlate with each other, as well as with depressive symptoms, has been reported. They also analyse correlations by correcting for guilt (when the effect excludes shame) or shame (when the effect includes guilt) and anxiety in addition to concentrating on bivariate associations.

\section{Association between shame, guilt and self-harm}

A subgroup of three studies more specifically measured self-harm than suicidal behaviour [6-7]. In those with a history of self-harm, two out of three studies showed significantly higher levels of shame than those without. In one psychiatric outpatient study [6], shame was not significantly correlated with self-harm frequency, and the trend direction was actually negative (greater guilt associated with less frequent selfharm), although the sample was very small, increasing the risk of unusual and unrepresentative results. Unpublished data also indicated a high level of guilt in the psychiatric patient.

The present article aimed at providing a systematic review of the available literature on self-harm and its relationship with shame and guilt. There was also evidence that there was a positive association between shame and suicidal behaviour and self-harm (where assessed as a general construct), but there were less studies and more diverse outcomes. On the other hand, the effects of the correlation between guilt and selfharm (including NSSI and suicidal behaviour) are mixed. Guilt proneness did not appear to be correlated with NSSI or suicidal behaviour, but the history of suicide attempts in four studies was associated with state guilt. While shame appears to be associated with self-harm, the absence of longitudinal studies limits conclusions about these associations ' direction or temporal characteristics.

The findings are mainly consistent with wider studies, where it has been found that shame has been positively associated with mental health problems, whereas the effects of remorse have been more uncertain $[2,8]$. The results show that elevated shame experiences are associated with self-harming behaviour. It is not possible to conclude that feelings of shame actively drive or maintain self-harm as the data are observational and correlation 
The data are consistent with emotion regulation-oriented model of self-harm, which view self-harm as a potential response to aversive affective states like shame [9-10], and with people's self-reported reasons for self-harm, that most commonly concern managing negative internal states including shame. However, shame experiences may also be a result of self-harm (e.g. scar-related self-injury shame;) [11], or an epiphenomenon related to other self-harming processes. As a way to control such self-directed feelings, selfharm can emerge. There is evidence that increased support for self-harm behaviour, shame-regulation purposes (i.e. reduction of shame) is associated with higher self-harm frequency [12].

\section{CONCLUSIONS}

It can be clearly concluded that shame has a positive association with self-harm whereas, guilt proneness does not appear to be related to self-harm, but caution should be taken in making further conclusions at this stage.

- Future research may benefit from moving beyond cross-sectional designs to better understanding this relationship.

- This study draws together the existing literature on shame, remorse, and self-harm. They provide a tentative indication of the nature and extent of the relationship between these (and their subtypes) emotions and self-harm.

- We also highlight important literature gaps and future directions, including the need for longitudinal designs and replications of earlier studies.

- Quantitative research may shed further light on the relationship between shame, guilt and self-harm.

\section{REFERENCES}

1. Sivasankari N, Shaiju B, Rahman J. A Study to Assess the Self-Harm Behaviours among Adolescents in a Selected University of Delhi with A View to Develop and Disseminate An Information Booklet on Prevention of Self-Harm Behaviours. Int J Sci Res 2016;5(2):1531-4.

2. Blythin SP, Nicholson HL, Macintyre VG, Dickson JM, Fox JR, Taylor PJ. Experiences of shame and guilt in anorexia and bulimia nervosa: A systematic review. Psychol Psychother Theory, Res Pract 2018;93(1):13459.

3. Brown MZ, Linehan MM, Comtois KA, Murray A, Chapman AL. Shame as a prospective predictor of selfinflicted injury in borderline personality disorder: A multi-modal analysis. Behav Res Ther 2009;47(10):81522.

4. Tangney JP, Stuewig J, Mashek DJ. Moral emotions and moral behavior. Ann Rev Psychol 2007;58:345-72.

5. Hasking P, Whitlock J, Voon D, Rose A. A cognitive-emotional model of NSSI: Using emotion regulation and cognitive processes to explain why people self-injure. Cogn Emot 2017;31(8):1543-56.

6. Lamb CS. Shame and self harm in Axis II disorders. Doctoral thesis: University of Edinburgh; 2004.

7. Milligan RJ, Andrews B. Suicidal and other self- harming behaviour in offender women: The role of shame, anger and childhood abuse. Legal Crim Psychol 2005;10(1):13-25.

8. Carden LJ, Saini P, Seddon C, Watkins M, Taylor PJ. Shame and the psychosis continuum: A systematic review of the literature. Psychol Psychother Theory Res Pract 2018;93(1):160-86.

9. Chapman AL, Gratz KL, Brown MZ. Solving the puzzle of deliberate self-harm: The experiential avoidance model. Behav Res Ther 2006;44(3):371-94.

10. Nock MK. Why do people hurt themselves? New insights into the nature and functions of self-injury. Curr Dir Psychol Sci 2009;18(2):78-83.

11. Bachtelle SE, Pepper CM. The physical results of nonsuicidal self-injury: The meaning behind the scars. J Nerv Ment Dis 2015;203(12):927-33.

12. Schoenleber $M$, Berenbaum H. Shame regulation in personality pathology. J Abnorm Psychol 2012;121(2):433-9.

Acknowledgements - Nil

Conflict of Interest - Nil; Funding - Nil

Indian Journal of Mental Health 2020;7(1) 